\title{
Acute Relationship between Cognitive and Psychological Symptoms of Patients with Mild Traumatic Brain Injury
}

\author{
Elaine de Guise, ${ }^{1,2}$ Joanne LeBlanc, ${ }^{1}$ Simon Tinawi, ${ }^{3}$ Julie Lamoureux, ${ }^{4}$ and Mitra Feyz ${ }^{1}$ \\ ${ }^{1}$ Traumatic Brain Injury Program, McGill University Health Centre-Montreal General Hospital, Montreal, QC, Canada H3G 1 A4 \\ ${ }^{2}$ Neurology and Neurosurgery Department, McGill University, Montreal, QC, Canada H3G 1A4 \\ ${ }^{3}$ Rehabilitation Medicine Department, McGill University Health Centre-Montreal General Hospital, Montreal, \\ QC, Canada H3G $1 A 4$ \\ ${ }^{4}$ Social and Preventive Medicine Department, University of Montreal, Montreal, QC, Canada H3C 3J7
}

Correspondence should be addressed to Elaine de Guise, elaine.deguise@muhc.mcgill.ca

Received 14 November 2011; Accepted 13 December 2011

Academic Editor: G. Kerkhoff

Copyright (c) 2012 Elaine de Guise et al. This is an open access article distributed under the Creative Commons Attribution License, which permits unrestricted use, distribution, and reproduction in any medium, provided the original work is properly cited.

\begin{abstract}
Objective. The goal of this study was to explore the relationship between acute psychological reactions and cognition as well as postconcussive symptoms in patients with MTBI. Research Methods. Sociodemographic and medical history data were gathered for 59 patients diagnosed with MTBI. Validated and standardized tools were used to assess anxiety, depression, and cognitive function two weeks after trauma. Postconcussive symptoms were assessed with the Rivermead postconcussive questionnaire. Results. Despite the absence of significant neuropsychological deficits, a very high level of anxiety and depression was observed in our cohort. Level of anxiety and depression were positively related to cognitive performances and to postconcussive symptoms. Moreover, patients with preexisting alcohol and psychological problems were more likely to present with acute depression after MTBI. Conclusions. Early psychological rehabilitation should be provided to decrease the intensity and frequency of postconcussive symptoms and diminish the risk of these problems becoming chronic.
\end{abstract}

\section{Introduction}

The incidence of mild traumatic brain injury (MTBI) is estimated at 100-300/100,000 population per year, representing 70 to 90 percent of all TBIs [1]. This constitutes a substantial number which creates significant pressure on the health system, as considerable time and resources are devoted to the assessment and rehabilitation of these patients by various health specialists. A multitude of symptoms are associated with MTBI, and appropriate intervention should be provided to decrease the frequency and duration of these.

Postconcussion syndrome (PCS) includes cognitive, emotional, and physical symptoms that some patients experience following MTBI or concussion. The WHO task force summarized the results of several studies on selfreported symptoms after MTBI. The most common symptoms reported after an MTBI were headache, blurred vision, dizziness, subjective memory problems, and other cognitive impairment as well as sleep problems [2]. In most patients, symptoms typically associated with MTBI improve quickly in the first days after the injury and largely resolve over the three-month period following the trauma [3, 4]. Many hypotheses focusing on different medical, psychological, and social issues have been entertained to explain why and who amongst the MTBI population is likely to endure long-term or even permanent symptoms. Among predictive factors of poorer recovery, the presence of past psychiatric problems [3], drug and alcohol abuse [5], financial incentives [6], and intensity of the acute symptomatology [7] are the variables most frequently cited. Furthermore, psychological reactions are one of the most prevalent complications following MTBI and tend to foster the maintenance of postconcussive symptoms [3].

The most common psychiatric sequelae of TBI include depression and anxiety [8]. The prevalence of depression after TBI is very high and estimated to be between $25 \%$ and 
$60 \%$ [9], whereas the prevalence of anxiety is estimated at $60 \%$ [8]. It is likely that anxiety and depression can arise directly or indirectly from the biological consequences of the TBI. This emotional status after TBI can be a psychological reaction to deficits and problems associated with having a brain injury or a reaction to multiple life stressors. More specifically, it could be a secondary response to failure to perform daily activities [10], maladaptive coping styles [11], or preinjury factors including vocational problems, lower education, and psychosocial difficulties [12]. It could also be a part of a preexisting mood disorder [3].

It is very difficult for clinicians to determine if a patient's self-reported symptoms are due to depression or to an anxiety disorder, PCS, or both [13]. Some studies have shown a positive relationship between the presence of depressed mood and anxiety with subjective cognitive complaints [14, 15]. Moreover, a strong relationship was observed between depression and cognitive impairment in patients with mildto-moderate TBI and preinjury psychiatric disorder such as depression and substance abuse $[5,6]$. Moreover, six months after trauma, self-reported cognitive complaints were more strongly related to premorbid traits and physical and emotional state factors than actual cognitive impairments [16]. Thus, it seems that there exists a discrepancy between the severity of cognitive complaints and the seemingly intact cognitive performances observed on neuropsychological assessment. These studies carried out in the later phases of recovery after trauma have demonstrated the complex interaction between PCS complaints, psychological status and cognitive functioning assessed via formal neuropsychological testing.

Little research has been carried out on the complex interaction of PCS symptoms, psychological reactions, and cognitive functioning in the early recovery phase with patients victim of MTBI. Knowing that PCS symptoms disappear in the first weeks and months for the majority of patients with MTBI, less medical attention and followup are provided to this population, and these patients rarely go through a complete formal medical, cognitive, and psychological assessment, even those who present with symptoms. These patients are not usually formally assessed in neuropsychology with a standard cognitive battery in the acute stage. Short screening tools and self-assessment of symptoms with questionnaires may be done acutely. To our knowledge, no studies have looked at the relationship between acute psychological and cognitive status (at two weeks after trauma) of patients with MTBI victims of general trauma using a standardized psychological and cognitive battery and taking into consideration the subjective cognitive, psychological, and physical complaints of these patients.

The goal of the present study was to measure acutely, via formal neuropsychological assessment, the postconcussive complaints and the psychological and cognitive status of patients with MTBI. More precisely, this study aimed to explore the associations between levels of anxiety and depression, visual integration, visual and sustained attention, speed of processing, selective attention and working memory, visual and verbal memory, verbal fluency, and arithmetic abilities as well as mental flexibility within the framework of psychological and cognitive processes. Validated and standardized psychological and cognitive tools as well as a PCS questionnaire were administered to a sample of patients with MTBI seen at an outpatient clinic two weeks after injury. Demographic and clinical variables were analysed as well as the interaction between premorbid status of the patients (history of substance abuse, preexisting psychological condition ...) and their psychological and PCS symptom status two weeks after MTBI. Based on studies done during the later recovery stage of patients with MTBI, we hypothesized that patients with high anxiety or depression levels would display lower scores on cognitive functions and more PCS complaints. Moreover, patients with pre-existing substance abuse and psychological antecedents would show a greater probability of developing acute psychological distress after MTBI.

Better knowledge of the acute psychological and cognitive status of patients with MTBI will allow clinicians and administrators to improve organization of acute rehabilitation services to this population and target specialized services acutely which could lead to a decrease in duration of symptoms as well as help decrease development of a chronic condition.

\section{Method}

2.1. Participants and Procedure. Along with a history of recent trauma to the head, identification of an MTBI was confirmed by a physician based on the WHO Task Force Criteria $[17,18]$. Operational criteria for clinical identification include 1 or more of the following: confusion or disorientation, loss of consciousness for 30 minutes or less, posttraumatic amnesia for less than 24 hours, and other transient neurological abnormalities such as focal signs, seizure, and intracranial lesion not requiring surgery. The duration of posttraumatic amnesia was assessed via a structured interview with the MTBI patient as well as from gathering information from the intake and progress notes of the emergency room nurses. Patients who had a posttraumatic amnesia of more than 24 hours were not considered as having an MTBI and were not included in the present study. The other inclusion criterion was a Glasgow Coma scale (GCS) score of 13-15 30 minutes after injury or later upon presentation for health care. The mechanism of injury included motor vehicle crash, falls, assaults, recreational sports accidents, and suicide attempts. In this latter group, only patients who had sustained a blunt trauma to the head were included and not those whose injury was hypoxic in nature.

Fifty nine patients diagnosed with MTBI who presented with postconcussive cognitive, psychological, or physical symptoms were referred to the ambulatory outpatient clinic from the emergency department and were seen for a followup between March 2006 and January 2007. They were assessed through a standardized evaluation protocol. An inhouse questionnaire ("Classification and Action Plan for 
MTBI") was used by the emergency physician as a systematic tool for referral of patients with MTBI to the clinic.

\subsection{Measures}

2.2.1. Previous Sociodemographic and Clinical Variables. Data was collected from medical charts and from semistructured interviews with the patient, the family, or the family physician. The variables considered were age at trauma, GCS scores, mechanism of the accident, gender, education, marital status, and working status. History of alcohol abuse was documented based on the classification of Corrigan and collaborators in 2005 [19]. Patients included in the group of preinjury alcohol abuse had heavy alcohol consumption (more than 14 drinks a week for men, and more than 7 drinks a week for women) at least in the last month prior to the TBI; this was confirmed by the patient, family member, or a relative and/or by the patient's family physician. Patients in the psychological antecedents group presented a clear psychiatric diagnosis based on DMS-IV. Another group of patients were classified according to medical antecedents. Patients with a history of one or more medical problems described in their hospital chart or discovered by semistructured interview were included in this group. This semistructured interview also included questions on the presence of previous TBI. Approval for this retrospective study was granted by the research ethics board of the MUHC-MGH.

2.2.2. Postconcussive Symptoms. Symptoms assessment was performed using the Rivermead postconcussion questionnaire (RPQ) [20]. This widely used tool consists of a list of 16 postconcussive symptoms that are ranked from 0 (not experienced at all) to 4 (severe problem). The RPQ was done at two weeks after the accident.

2.2.3. Psychological Assessment. The psychological assessment included two tests to measure the level of anxiety and depression, the Beck anxiety scale [21], and the Beck depression scale [22] which were presented two weeks after TBI.

2.2.4. Cognitive Assessment. Visual integration was assessed with the Hooper visual integration test (HVIT) [23], and visual attention and visual neglect were assessed with the bells test looking at the time to circle 35 bells among other stimuli. The number of omissions and time to complete the task were recorded [24]. Sustained attention was assessed with the mental manipulation subtest of the WSM-III [25] and speed of processing with the symbols subtest of WAISIII [26]. The digit span subtest of the WSM-III [25] explored working memory. Visual memory was assessed with copy and immediate and delayed recall of the Rey-Osterrieth complex figure [27] and verbal learning with the Hopkins verbal learning test [28]. The controlled oral and word association (COWA) test looked at phonological and semantic fluency [29], while the arithmetic subtest of the WAIS-III [26] looked at arithmetic abilities. Finally, trail A and trail B tested mental flexibility [30].
An experienced neuropsychologist administered the psychological and cognitive measures.

2.3. Statistical Analyses. Descriptive statistics for baseline characteristics and all outcome variables were computed. Associations between numerical variables were assessed with Pearson correlation coefficients. Associations involving ordinal variables were assessed with Spearman rank correlation coefficients. Simple comparisons of groups were done using $t$-tests or ANOVAs (for numerical variables in large groups), nonparametric Mann-Whitney $U$ or Kruskall-Wallis (for ordinal variables or numerical variables in small groups with nonnormal distributions), and chi-square tests (for nominal variables). Considering the large number of association measures calculated in this study and its exploratory nature, we opted for a level of significance of $P<0.01$ with the mention of the need for further investigation when the level of significance was found to be between 0.01 and 0.05 . All analyses were done using SPSS 17.0.

\section{Results}

3.1. Previous Sociodemographic and Clinical Variables. Mean age of those referred for neuropsychological assessment was 35.5 years $(\mathrm{SD}=12.24$, range $=16-81$, median $=35)$. Only one subject aged 81 years old, and all other subjects aged between 16 and 59 years of age. The mean score on the GCS was $14.59(\mathrm{SD}=0.67$, range $=13-15) .10 .2 \%$ of patients had a score of 13 , on the GCS, $20.3 \%$ had a score of 14 , and the majority of the group (69.5\%) had a score of 15 . Given that 18 out of the 59 subjects (30.5\%) in this study had a history of psychological antecedents (13 depression, 3 anxiety, 1 bipolar, and 1 psychosis), all results for this group will be compared to the group without pretrauma psychological problems. There was no difference in age or in GCS between the groups with and without psychological antecedents.

The mechanism of injury included motor vehicle crashes (49.2\%), falls $(20.3 \%)$, assaults $(8.5 \%)$, recreational sports accidents (1.6\%), and suicide attempts (3.4\%). The demographic and pre-trauma clinical variables of the 59 patients with MTBI seen for a neuropsychological assessment are presented in Table 1. Although the mechanism of trauma did not differ significantly between the groups with and without psychological antecedents, $\left(\chi_{4 \mathrm{df}}^{2}=7.187, P=0.126\right)$, both suicide attempts in this sample were carried out by subjects with psychological antecedents of depression.

3.2. Postconcussive Symptoms Analysis. Table 2 gives the frequency distribution of the various symptoms measured by the RPQ. Certain symptoms were more common in this group of subjects: $75 \%$ of the sample complained of moderate-to-severe fatigue, $63 \%$ complained of moderateto-severe headaches, and $51 \%$ complained of moderate-tosevere problems in concentration. Some symptoms were very uncommon: double vision was absent in $68 \%$ of the sample, restlessness was absent in 56\%, and nausea and vomiting were absent in $53 \%$ of the sample. 
TABLE 1: Demographic and pretrauma clinical characteristics $(n=$ 59).

\begin{tabular}{|c|c|c|c|}
\hline Variable & Category & $N$ & $\%$ \\
\hline \multirow{2}{*}{ Gender } & Male & 34 & 57.6 \\
\hline & Female & 25 & 42.4 \\
\hline \multirow{3}{*}{ Education (in years) } & $1-6$ yrs. & 0 & 0.0 \\
\hline & $7-13$ yrs. & 26 & 44.1 \\
\hline & $14+$ yrs. & 33 & 55.9 \\
\hline \multirow{4}{*}{ Marital status } & Single & 31 & 52.5 \\
\hline & Married & 18 & 30.5 \\
\hline & Divorced & 10 & 16.9 \\
\hline & Widowed & 0 & 0.0 \\
\hline \multirow{6}{*}{ Working status } & Manual labor & 9 & 15.3 \\
\hline & Technical services & 19 & 32.2 \\
\hline & Professional & 18 & 30.5 \\
\hline & Student & 9 & 15.3 \\
\hline & None & 3 & 5.1 \\
\hline & Retired & 1 & 1.7 \\
\hline \multirow{2}{*}{ Alcohol or drug history } & Yes & 9 & 15.3 \\
\hline & No & 50 & 84.7 \\
\hline \multirow{2}{*}{ Psychological history } & Yes & 18 & 30.5 \\
\hline & No & 41 & 69.5 \\
\hline \multirow{2}{*}{ Medical history } & Yes & 2 & 3.4 \\
\hline & No & 57 & 96.6 \\
\hline \multirow{2}{*}{ Previous TBI } & Yes & 12 & 20.3 \\
\hline & No & 47 & 79.7 \\
\hline
\end{tabular}

The comparative distribution of severity of symptoms between those with and without psychological antecedents was not significantly different except for item 8 of the RPQ (feeling depressed, $\chi_{4 \mathrm{df}}^{2}=20.705, P<0.001$ ) which was significantly more serious a symptom in the group with psychological antecedents.

Overall, the group with psychological antecedents had a marginally higher total score on the RPQ $(35.6 \pm 13.5$ versus $26.4 \pm 13.3, P=0.018)$ compared to the group without psychological antecedents.

\subsection{Psychological Symptoms}

3.3.1. Beck Depression Score. The values ranged from 6 to 39 with a mean $( \pm s d)$ of $17.61 \pm 8.72$. This sample displayed significantly higher scores from the normative sample described by Creamer and colleagues [31] $\left(t_{377 \mathrm{df}}=\right.$ $7.887, P<0.001)$. It should be noted that the comparative sample in this case was younger (average age of 20 years old) and composed mostly of females (74\%). In our sample, thirteen subjects $(22.0 \%)$ had a score between 1 and 10 (normal ups and downs), 22 (37.3\%) between 11 and 16 (mild mood disturbance), 6 (10.2\%) between 17 and 20 (borderline clinical depression), 11 (18.6\%) between 21 and 30 (moderate depression), and 7 (11.9\%) between 31 and 40 (severe depression). No subject had a score over 40 which corresponds to extreme depression. According to normative data from Pomerantz and colleagues [32], our sample

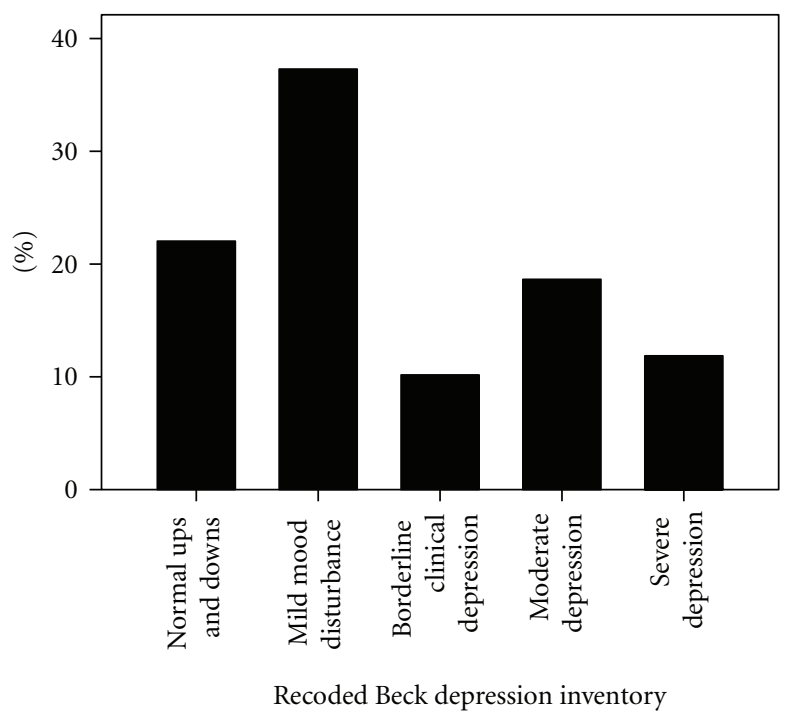

Figure 1: Distribution of depression categories $(n=59)$.

had significantly more subjects with moderate depression compared to a normal population $\left(\chi_{3 \mathrm{df}}^{2}=10.613, P=\right.$ 0.014 ). Figure 1 gives the distribution of these five categories of depression in our sample. The average depression score was significantly higher in the group with psychological antecedents $(24.7 \pm 7.8$ versus $14.5 \pm 7.2, P<0.001)$.

3.3.2. Beck Anxiety Score. The values on the Beck anxiety score ranged from 10 to 38 with a mean $( \pm s d)$ of $26.61 \pm$ 6.97. This sample displayed significantly higher scores from the normative sample described by Creamer and colleagues [31] $\left(t_{382 \mathrm{df}}=10.323, P<0.001\right)$. It should be noted that the comparative sample in this case was younger (average age of 20 years old) and composed mostly of females (74\%). In our sample, twelve subjects $(20.3 \%)$ had a score between 0 and 21 (very low anxiety), 43 (72.9\%) had a score between 22 and 35 (moderate anxiety), and $4(6.8 \%)$ had a score that exceeded 36 (potential cause for concern) (see Figure 2). According to normative data from Creamer and colleagues [31], our sample had significantly more subjects with moderate anxiety compared to a normal population $\left(\chi_{2 \mathrm{df}}^{2}=7.870, P=0.020\right)$. The average anxiety score was significantly higher in the group with psychological antecedents $(32.0 \pm 5.3$ versus $24.2 \pm 6.3, P<0.001)$.

3.4. Correlation between Anxiety, Depression, and Demographic/Pretrauma Clinical Variables. There was a moderate correlation $(r=0.539, P<0.001)$ between the anxiety and the depression scores. Those who had a higher anxiety score also had a higher depression score (see Figure 3). When comparing those with and without psychological antecedents, the correlation remained significant in the latter group $(r=0.523, P<0.001)$ but was not significant in the group with psychological antecedents $(r=-0.061, P=$ $0.811)$. Neither the depression $\left(r_{\text {Spearman }}=0.235, P=\right.$ $0.073)$ nor the anxiety score $\left(r_{\text {Spearman }}=0.188, P=0.153\right)$ was associated with age. Likewise, neither the depression 


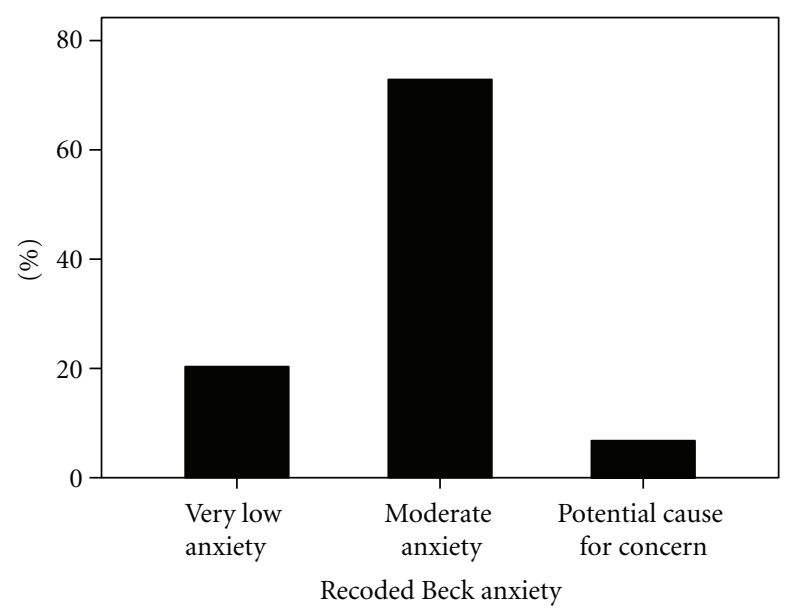

Figure 2: Distribution of anxiety categories $(n=59)$.

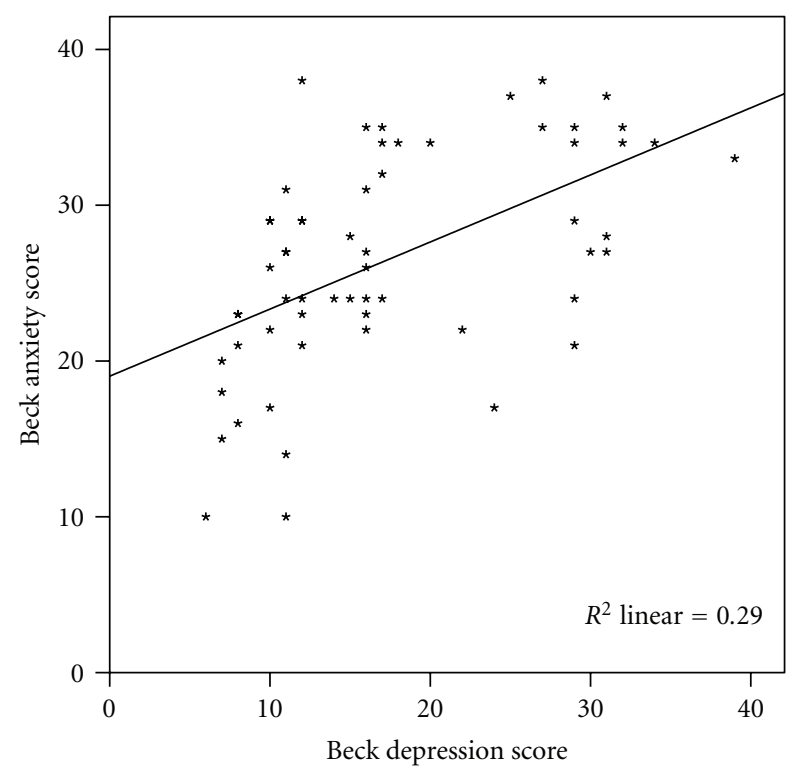

Figure 3: Correlation between anxiety and depression scores $(n=$ 59).

$\left(r_{\text {Spearman }}=-0.241, P=0.066\right)$ nor the anxiety score $\left(r_{\text {Spearman }}=0.103, P=0.436\right)$ weas associated with the initial GCS score.

The Beck scores (both anxiety and depression) were not significantly different between men and women or between subjects of different education levels. Both the depression scores $\left(F_{(2,72 \mathrm{df})}=9.555, P<0.001\right)$ and the anxiety scores $\left(F_{(2,72 \mathrm{df})}=4.693, P=0.012\right)$ were significantly higher for the divorced group compared to the single or married group. The anxiety score was significantly higher for those who were working before the trauma $\left(F_{(1,73 \mathrm{df})}=\right.$ 5.785, $P=0.019$ ) compared to those who were not working or who were students, whereas the depression scores were not significantly different between these two groups. The anxiety scores were not significantly different between the groups with and without alcohol or drug antecedents, whereas the depression scores were significantly higher in those with such antecedents $\left(t_{(73 \mathrm{df})}=2.965, P=0.004\right)$. Both the depression scores $\left(t_{(73 \mathrm{df})}=5.272, P<0.001\right)$ and the anxiety scores $\left(t_{(73 \mathrm{df})}=4.095, P<0.001\right)$ were higher in the group with psychological antecedents compared to the group without such antecedents. There was no significant difference between the groups with and without medical antecedents or the groups with or without a previous TBI for either Beck scores.

3.5. Cognitive Analysis. Table 3 provides descriptive statistics for all neuropsychological tests.

3.5.1. Visual Integration. Scores on the HIVT ranged from 7 to 30 with a mean $( \pm s d)$ of $27.66 \pm 3.13$. Fifty six subjects (94.9\%) had a score between 24 and 30 (very low probability of impairment), 2 (3.4\%) had a score between 21 and 23 (low probability of impairment), and $1(1.7 \%)$ had a score lower than 16 (very high probability of impairment). Not having access to a normal distribution of these scores, it is unclear how the results compare to a normal population. There was no significant differences between the group with $(27.3 \pm 2.2)$ and without $(27.8 \pm 3.5)$ psychological antecedents $\left(t_{57 \mathrm{df}}=\right.$ $0.529, P=0.599)$.

3.5.2. Visual Attention. Only the total number of omissions on the bell test was analysed as completion time of the bell test is not considered to be a valid indicator of success or neglect as normal subjects take between 1 and 5 minutes to complete it with no omissions [24]. Scores were very skewed and ranged from 0 to 15 minutes with a mean $( \pm s d)$ of $2.81 \pm 3.51$ (median $=2$ ). In the literature, the number of omissions is reported according to the side of the page where the omission was made, and this is related to the laterality of the brain damage. Since the norms are the same for right and left errors in the normal population, right-side norms were used. Seventeen subjects $(28.8 \%)$ of the MTBI sample had no omissions, $24(30.7 \%)$ had 1 to 3 omissions, and 18 $(30.5 \%)$ had 4 omissions or more. The chi-square comparing the distribution of normal subjects with that of the group with mTBI was marginally significant $\left(\chi_{2 \mathrm{df}}^{2}=9.058, P=\right.$ $0.011)$. Subanalyses indicated that the probability of having 4 or more omissions in this MTBI population is significantly higher than in the normal population. It is interesting to note that the group with psychological antecedents also showed a tendency for more omissions without reaching statistical significance (on average $4.1 \pm 3.8$ versus $2.2 \pm 3.3, P=0.059$ ).

3.5.3. Sustained Attention. Scores on the mental manipulation subtest ranged from 14 to 40 with a mean $( \pm s d)$ of $24.22 \pm 6.80$ (Median $=25.0$ ) and were compared to the percentile ranks for a normative population. There were only 2 subjects $(3.4 \%)$ with a score lower than the 25 th percentile and 1 subject $(1.7 \%)$ with a score higher than the $75 \%$ percentile. Since the percentages of subjects in extreme quartiles were much lower than $25 \%$, we cannot conclude that our sample had scores significantly higher or lower than the norms, but the distribution of cases was significantly 
TABLE 2: Symptoms of the Rivermead postconcussive questionnaire $(n=59)$.

\begin{tabular}{|c|c|c|c|c|c|}
\hline Items & $\begin{array}{l}\text { Not experienced } \\
\text { at all }\end{array}$ & $\begin{array}{c}\text { Not a } \\
\text { problem } \\
\text { anymore }\end{array}$ & $\begin{array}{c}\text { Mild } \\
\text { problem }\end{array}$ & $\begin{array}{l}\text { Moderate } \\
\text { problem }\end{array}$ & $\begin{array}{c}\text { Severe } \\
\text { problem }\end{array}$ \\
\hline (1) Headaches & $9(15.3)$ & $4(6.8)$ & $9(15.3)$ & $23(39.0)$ & $14(23.7)$ \\
\hline (2) Feelings of dizziness & $11(18.6)$ & $5(8.5)$ & $18(30.5)$ & $16(27.1)$ & $9(15.3)$ \\
\hline (3) Nausea and or vomiting & $31(52.5)$ & $5(8.5)$ & $15(25.4)$ & $5(8.5)$ & $3(5.1)$ \\
\hline (4) Noise sensitivity & $18(30.5)$ & $5(8.5)$ & $15(25.4)$ & $13(22.0)$ & $8(13.6)$ \\
\hline (5) Sleep disturbance & $18(30.5)$ & $5(8.5)$ & $8(13.6)$ & $15(25.4)$ & $13(22.0)$ \\
\hline (6) Fatigue & $3(5.1)$ & $1(1.7)$ & $11(18.6)$ & $21(35.6)$ & $23(39.0)$ \\
\hline (7) Irritability & $17(28.8)$ & $3(5.1)$ & $14(23.7)$ & $13(22.0)$ & $12(20.3)$ \\
\hline (8) Feeling depressed & $25(42.4)$ & $6(10.2)$ & $11(18.6)$ & $10(16.9)$ & $7(11.9)$ \\
\hline (9) Feeling frustrated & $15(25.4)$ & $4(6.8)$ & $14(23.7)$ & $14(23.7)$ & $12(20.3)$ \\
\hline (10) Forgetfulness, poor memory & $8(13.6)$ & $5(8.5)$ & $21(35.6)$ & $16(27.1)$ & $9(15.3)$ \\
\hline (11) Poor concentration & $7(11.9)$ & $0(0.0)$ & $22(37.3)$ & $14(23.7)$ & $16(27.1)$ \\
\hline (12) Taking longer to think & $11(18.6)$ & $4(6.8)$ & $18(30.5)$ & $14(23.7)$ & $12(20.3)$ \\
\hline (13) Blurred vision & $20(33.9)$ & $11(18.6)$ & $22(37.3)$ & $6(10.2)$ & $0(0.0)$ \\
\hline (14) Light sensitivity & $23(39.0)$ & $8(13.6)$ & $13(22.0)$ & $12(20.3)$ & $3(5.1)$ \\
\hline (15) Double vision & $40(67.8)$ & $10(16.9)$ & $8(13.6)$ & $1(1.7)$ & $0(0.0)$ \\
\hline (16) Restlessness & $33(55.9)$ & $2(3.4)$ & $10(16.9)$ & $10(16.9)$ & $4(6.8)$ \\
\hline
\end{tabular}

TABLE 3: Descriptive statistics for the neuropsychological assessment $(n=59)$.

\begin{tabular}{lccc}
\hline Tests & Mean & SD & Range \\
\hline Hooper (raw score) & 27.66 & 3.13 & $8-30$ \\
Bell (omissions) & 2.81 & 3.51 & $0-15$ \\
Mental manipulation (raw score) & 24.22 & 6.80 & $14-40$ \\
Symbols (raw score) & 67.56 & 19.74 & $4-103$ \\
Digit forward (raw score) & 6.24 & 1.16 & $4-9$ \\
Digit backward (raw score) & 4.78 & 1.21 & $3-8$ \\
Rey immediate (raw score) & 22.78 & 7.17 & $4-34$ \\
Rey delayed (raw score) & 20.58 & 6.76 & $2-32$ \\
Hopkins R1 (raw score) & 6.97 & 1.72 & $3-11$ \\
Hopkins R2 (raw score) & 9.44 & 2.03 & $3-12$ \\
Hopkins R3 (raw score) & 10.25 & 2.21 & $3-12$ \\
Hopkins delayed (raw score) & 8.98 & 2.82 & $1-12$ \\
Hopkins recognition (raw score) & 11.71 & 2.53 & $5-28$ \\
Phonological fluency (raw score) & 13.37 & 4.43 & $6-27$ \\
Semantic fluency (raw score) & 20.22 & 5.08 & $9-34$ \\
Arithmetic (raw score) & 14.66 & 4.46 & $7-28$ \\
Trail A (in seconds) & 32.32 & 16.14 & $8-103$ \\
Trail B (in seconds) & 69.39 & 30.23 & $30-181$ \\
\hline
\end{tabular}

more homogeneous, in regards to sustained attention, than the normative data $\left(\chi_{2 \mathrm{df}}^{2}=50.844, P<0.001\right)$, that is, significantly more subjects were near the average score in our sample. The group with psychological antecedents had a significantly lower score on the mental manipulation subtest than the group without $(19.8+6.4$ versus $26.1 \pm 6.1, P=$ $0.001)$.
3.5.4. Speed of Processing. The values for the symbols subtest scores ranged from 4 to 103 with a mean $( \pm \mathrm{sd})$ of 67.56 \pm 19.74 (Median $=72.0$ ) and were compared to percentile ranks for a normal population. There were only 5 subjects (8.5\%) with a score lower than the 25th percentile and no subject with a score higher than the $75 \%$ percentile. Since the percentages of subjects in extreme quartiles were much lower than $25 \%$, we cannot conclude that our sample had scores significantly higher or lower than the norms but, again, the distribution for speed of processing was significantly more homogeneous than the normative data $\left(\chi_{2 \mathrm{df}}^{2}=41.601, P<\right.$ $0.001)$, that is, significantly more subjects were near the average score in our sample. Marginally, the group with psychological antecedents showed a tendency for lower scores on the symbol test than the group without such antecedents $(57.8 \pm 23.6$ versus $71.8 \pm 16.4, P=0.011)$.

3.5.5. Selective Attention and Working Memory. The values of the digit forward test ranged from 4 to 9 with a mean $( \pm s d)$ of $6.24 \pm 1.16$. (median $=6.0)$. The values of the digit backward test ranged from 3 to 8 with a mean $( \pm \mathrm{sd})$ of $4.78 \pm 1.21$ (median $=5.0)$. These scores were not significantly different from the normative scores. The group with psychological antecedents had lower scores on the digit forward test than the group without such antecedents $(5.6 \pm 1.0$ versus $6.5 \pm 1.1$, $P=0.002)$.

3.5.6. Visual Memory. Results on the Rey-Osterrieth complex figure tests for this sample were compared with normative data according to age group. It appears that the results of our cohort were not significantly different from the norms, keeping in mind that the subsamples were very 
small (between 3 and 12) which diminishes substantially the power of the comparisons. The groups with and without psychological antecedents were not significantly different on the Rey tests.

3.5.7. Verbal Memory. Data for the Hopkins verbal learning test were compared to norms in the case of immediate recall. In this sample, the mean \pm sd was $6.97 \pm 1.72($ median $=7.0)$, ranging from 3 to 11 , compared to the normative values of $7.17 \pm 2.18$. The difference was not statistically significant $(P=0.661)$. The groups with and without psychological antecedents did not have significantly different results in the immediate recall test (resp., $6.5 \pm 2.1$ and $7.2 \pm 1.5, P=0.170$ ), but the group with psychological problems pretrauma did show a tendency for lower scores at the 3rd recall $(9.1 \pm 2.9$ versus $10.8 \pm 1.6, P=0.026)$.

3.5.8. Verbal Fluency. Results on the COWA test for this sample were compared to norms according to levels of schooling. The educational level categories used in this research were not, however, exactly the same as the categories referred to in the norms. The high school subgroup of subjects $(n=13)$ were compared to the normative data for 9 to 12 years of schooling $(n=479)$. The subjects with MTBI had a mean $( \pm s d)$ of $13.06 \pm 4.39$ for the phonological fluency test compared to $13.7 \pm 4.5$. The difference was not statistically significant $(P=0.575)$. The post-high-school subgroup $(n=15)$ were compared to the normative data for 13 to 16 years of schooling $(n=332)$. The subjects with MTBI had a mean $( \pm \mathrm{sd})$ of $13.77 \pm 4.44$ for the phonological fluency test compared to $15.1 \pm 4.1$. The difference was not statistically significant $(P=0.183)$. The university subgroup $(n=31)$ were compared to the normative data for 17 to 21 years of schooling $(n=51)$. The subjects with MTBI had a mean $( \pm \mathrm{sd})$ of $13.23 \pm 4.48$ on the phonological fluency test compared to $16.2 \pm 4.1$. In this case, the difference was statistically significant $(P=0.003)$. Phonological fluency was also significantly lower in the group with psychological antecedents compared to the group without ( $10.3 \pm 3.4$ versus $14.7 \pm 4.2, P<0.001)$.

With regards to semantic fluency on this test, data were compared to norms according to age groups. The results indicated that the TBI group was not significantly different from the normal group.

3.5.9. Arithmetic Abilities. The values for arithmetic scores ranged from 7 to 28 with a mean ( \pm sd) of $14.66 \pm$ 4.46 (median $=15.0)$, and these results were compared to percentile ranks for a normal population. There were only 2 subjects $(3.4 \%)$ with a score lower than the 25 th percentile and 3 subjects $(5.1 \%)$ with a score higher than the 75 th percentile. Since the percentages of subjects in extreme quartiles were much lower than $25 \%$, we could not conclude that our sample had scores significantly higher or lower than the norms, but the distribution of cases was significantly more homogeneous than the normative data $\left(\chi_{2 \mathrm{df}}^{2}=43.042, P<0.001\right)$, that is, significantly more subjects were near the average score in our sample. The results for arithmetic abilities were significantly lower in the group with psychological antecedents compared to the group without $(11.2 \pm 3.0$ versus $16.2 \pm 4.1, P<0.001)$.

3.5.10. Mental Flexibility. Results on the trail making test for this sample were compared to norms by age groups which were further divided by levels of education. The values for trail making A ranged from 8 to 103 with a mean $( \pm \mathrm{sd})$ of $32.32 \pm 16.14$ (Median = 29.0). The values for trail making $B$ ranged from 30 to 181 with a mean $( \pm s d)$ of $69.39 \pm 30.23$ $($ median $=61.0)$. None of comparisons by age reached the level of significance of 0.01 . There was a tendency for the group with psychological antecedents to have a higher score (longer reaction time) on the trail making $\mathrm{B}(81.6 \pm 35.7$ versus $64.1 \pm 26.2, P=0.039$ ) compared to the group without psychological antecedents.

3.6. Correlation among Anxiety, Depression, and Cognitive Performances. The Pearson correlation coefficients of the Beck depression and Beck anxiety scores with the neuropsychological assessments are given in Table 4. Sixteen out of the 20 cognitive tests were correlated to the Beck depression score, and 14 out of the 20 were correlated with the Beck anxiety score showing a clear relationship among depression, anxiety, and cognition in the acute phase of recovery.

\subsection{Correlation between the Rivermead Postconcussion Ques-} tionnaire Scores and Cognitive Performances. The correlation coefficients (Kendall tau) are presented in Table 5. The correlations between the psychometric test results and the scores of the RPQ were all inferior to 0.4 which means that the associations between the cognitive/psychological measures and the postconcussive symptoms were weak. Generally speaking, the Beck depression scale and the trail A were the two tests most closely associated with most of the items of the RPQ (12 out of 16 items), followed by the Beck anxiety inventory, the mental manipulation test, the digit span forward, and digit span backward (all with 8 items out of 16). Some tests were not significantly associated with any of the items of the RPQ (delayed subtest of the Hopkins verbal learning test and phonological fluency). The RPQ items that showed the highest number of significant correlation coefficients with neuropsychological tests were item 8 (feeling depressed) which was associated with 9 tests and items 5 (sleep disturbance) and 6 (fatigue) that were associated with 7 tests.

\section{Discussion}

The goal of the present study was to explore, via a formal neuropsychological assessment, the relationship between postconcussive complaints, acute psychological status, and cognitive status of patients with mTBI. Results obtained were compatible with the hypothesis proposed. A significant relationship was obtained between the level of anxiety and depression of patients with MTBI and their cognitive performances as well as with their PCS complaints (the higher the depression or anxiety scores, the more severe 
TABle 4: Correlation coefficients between the Beck scores and neuropsychological tests $(n=59)$.

\begin{tabular}{|c|c|c|}
\hline Tests & Beck depression score & Beck anxiety score \\
\hline Hooper & -.137 & -.160 \\
\hline Sig & .301 & .226 \\
\hline Bell: completion time & $.283^{*}$ & $.322^{*}$ \\
\hline Sig & .030 & .013 \\
\hline Bell: nb omissions & $.409^{* *}$ & $.318^{*}$ \\
\hline Sig & .001 & .014 \\
\hline Mental manipulation & $-.579^{* *}$ & $-.262^{*}$ \\
\hline Sig & .000 & .045 \\
\hline Symbols & $-.373^{* *}$ & $-.305^{*}$ \\
\hline Sig & .004 & .019 \\
\hline Digit span forward & $-.402 * *$ & $-.347^{* *}$ \\
\hline Sig & .002 & .007 \\
\hline Digit span backward & -.115 & -.073 \\
\hline Sig & .385 & .583 \\
\hline Rey: copy & -.254 & -.224 \\
\hline Sig & .052 & .088 \\
\hline Rey: immediate & $-.298^{*}$ & -.195 \\
\hline Sig & .022 & .138 \\
\hline Rey: delayed & $-.263^{*}$ & -.225 \\
\hline Sig & .044 & .086 \\
\hline Hopkins: R1 & $-.270^{*}$ & -.090 \\
\hline Sig & .039 & .497 \\
\hline Hopkins: R2 & $-.496^{* *}$ & $-.347^{* *}$ \\
\hline Sig & .000 & .007 \\
\hline Hopkins: R3 & $-.476^{* *}$ & $-.391^{* *}$ \\
\hline Sig & .000 & .002 \\
\hline Hopkins: delayed & $-.450 * *$ & $-.289^{*}$ \\
\hline Sig & .000 & .026 \\
\hline Hopkins: recognition & -.235 & -.245 \\
\hline Sig & .073 & .062 \\
\hline Phonological fluency & $-.476^{* *}$ & $-.328^{*}$ \\
\hline Sig & .000 & .011 \\
\hline Semantic fluency & $-.445^{* *}$ & $-.307^{*}$ \\
\hline Sig & .000 & .019 \\
\hline Arithmetics & $-.512 * *$ & $-.394^{* *}$ \\
\hline Sig & .000 & .002 \\
\hline Trail A & $.277^{*}$ & $.332^{*}$ \\
\hline Sig & .033 & .010 \\
\hline Trail B & $.335^{* *}$ & $.265^{*}$ \\
\hline Sig & .009 & .042 \\
\hline
\end{tabular}

${ }^{*} P<0.05,{ }^{* *} P<0.01$.

the complaints). Moreover, the patients with a preexisting alcohol and psychological history were more at risk of showing acute depression after MTBI. The relationship of these variables with anxiety problems was less significant.

4.1. Postconcussive Complaints and Psychological and Cognitive Profiles of Patients with MTBI. One of the goals of this investigation was to obtain an acute profile of PCS, psychological and cognitive symptoms in patients with MTBI. At two weeks after trauma, these patients complained mainly about moderate-to-severe fatigue $(75 \%)$, moderateto-severe headaches $(63 \%)$ and moderate-to-severe concentration problems $(51 \%)$ which is compatible with other studies [2]. Of note, $52.6 \%$ of them did not complain about depressed mood, but their scores were very high on the Beck depression scale. The majority of subjects obtained scores reflecting mild mood disturbance $(37.3 \%)$ with $10.2 \%$ for borderline clinical depression, $18.6 \%$ moderate depression, and $11.9 \%$ severe depression. Moreover, the majority of patients $(72.9 \%)$ showed measures of moderate anxiety, whereas $6.8 \%$ had a very high level of anxiety. However, in formal neuropsychological testing, no significant difference was found between norms and the results of our cohort on tests measuring visual integration, sustained attention, speed of processing, visual memory, semantic fluency, and arithmetic and mental flexibility. The only mild differences between norms and the group studied were for visual attention and phonological fluency. However, it should be pointed out that in comparing our sample with norms, numbers for subcategories of age or education became very small, reducing the power of statistical comparisons. A few studies have already shown no significant deficit in formal neuropsychological testing with this population even if patients had significant complaints [33]. This absence of significant cognitive deficit could be explained by the lack of sensitivity of the cognitive tests used in this investigation to the actual deficits experienced. Other evaluation methods such as electrophysiological and imaging studies have shown abnormalities in the trauma population despite a normal cognitive assessment [34]. Finally, the results of the present study would perhaps have been different if asymptomatic MTBI patients had been included; this retrospective investigation did not include the asymptomatic patients as they were not referred to the clinic. Thus, a patient selection bias may have influenced the profile of the sample. Including only symptomatic patients probably increased the postconcussive symptoms values as well as the anxiety and depression levels.

What was more concerning in the results of this study was the higher levels of depression and anxious mood in our cohort. It seems that there is a critical period of psychological distress after an MTBI which could influence the patient's perceptions of symptoms assessed by the RPQ and which is observed by the correlations between the level of anxiety and depression and the PCS symptoms. An acute psychological reaction after TBI may reflect a predisposition for this mTBI population to have a higher prevalence of depression and anxiety to begin with or a secondary response to failure to perform daily activities (e.g., not able to reintegrate work) or maladaptive coping styles, even very acutely after accident. These maladaptive reactions have been observed later in the process of recovery $[10,11]$, and this study has shown the possibility that a similar process is found acutely. However, it is unclear if the intensity of PCS symptoms serves to increase anxiety level and depression or if anxiety level and depression influences the severity of perception of symptoms. There is a growing body of literature that calls for caution in 


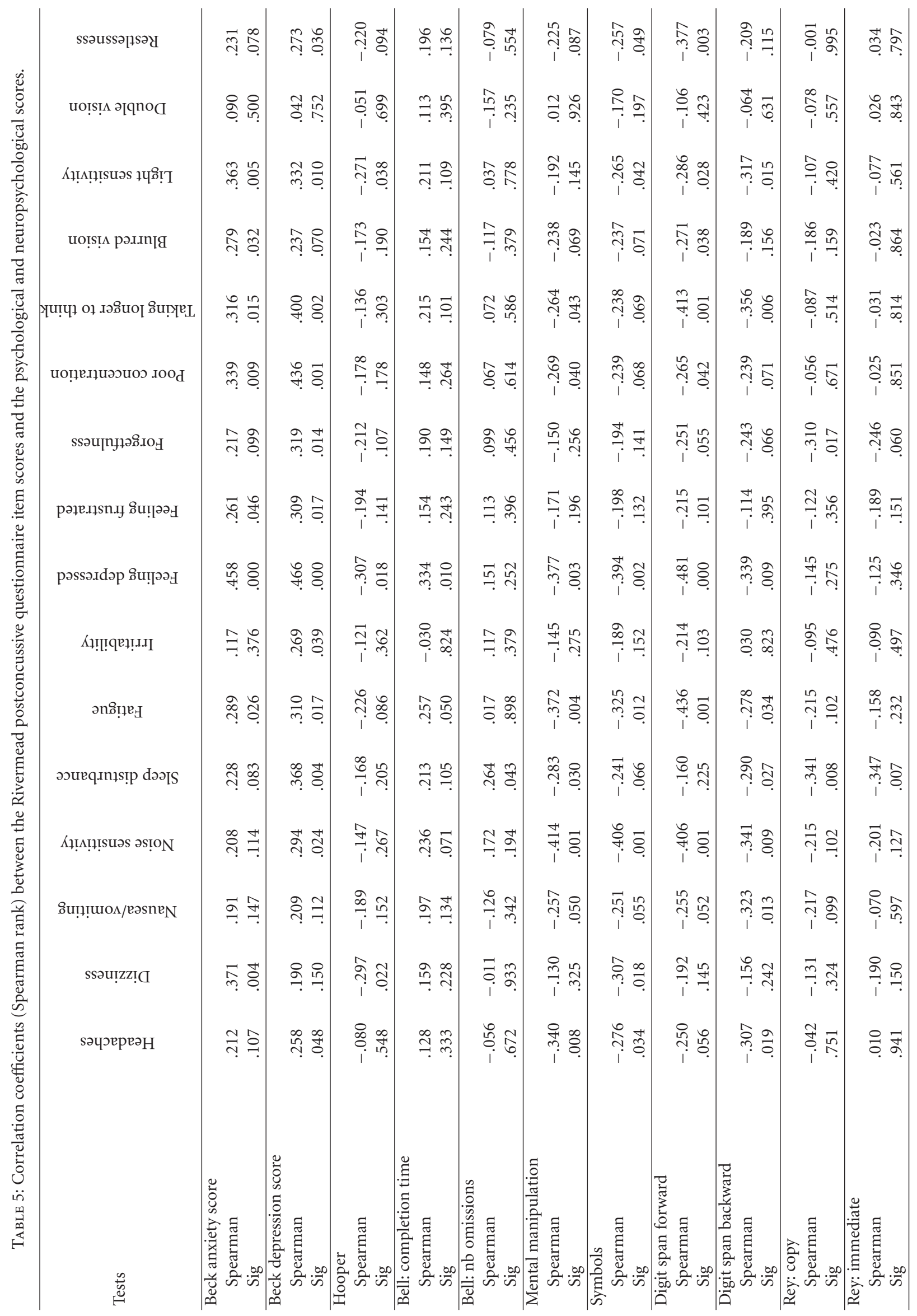




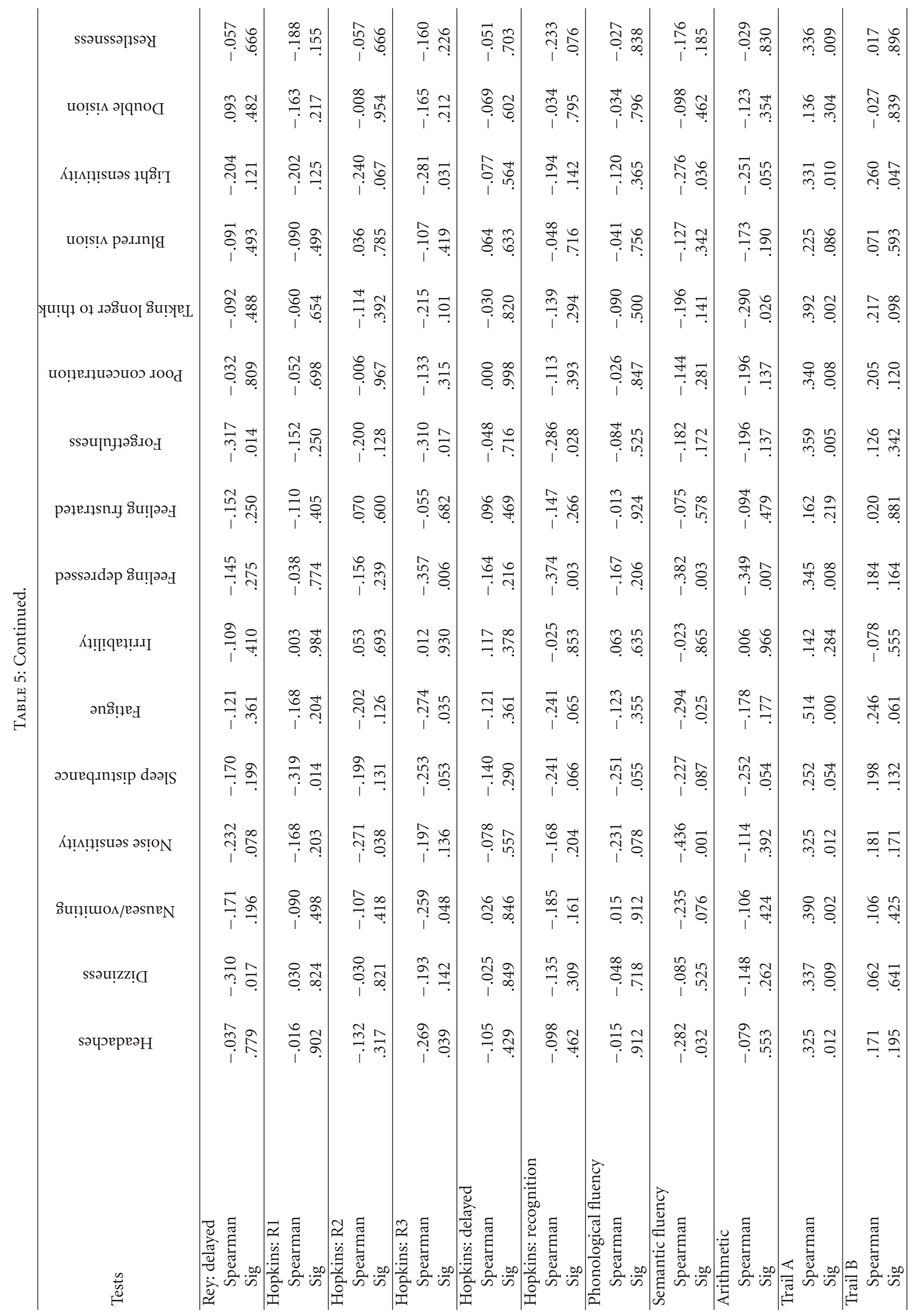


the clinical interpretation of results from PCS inventories. Garden and Sullivan [35] examined the relationship between PCS symptoms and depression measured by the Beck depression inventory in healthy subjects. They observed a strong positive relationship between depression and PCS, results that were consistent with the literature suggesting a significant role for nonneurological factors in the expression of PCS symptomatology. Moreover, it has been argued that the use of checklists for evaluating cognitive states results in overreporting, as they are nonspecific and sensitive to recall bias and strongly influenced by emotional distress [36, 37]. In the current study, we can only conclude that there is also a strong relationship between psychological distress and the perception of symptoms in the first two weeks after MTBI in a general trauma population. In addition, level of depression and cognitive performances were related which is in keeping with what has already been published in the literature for a depressed population $[38,39]$ and for a TBI population [40]. Furthermore, as in other research [41], a positive relationship between the level of anxiety and cognitive performances was also observed. Another variable that should be considered in this investigation which may have influenced the patient's level of psychological reaction is the possible addition of some symptoms related to posttraumatic stress disorder (PTSD) in patients who were involved in an assault. Only a small percentage of the sample was victims of assault (8.5\%), and these patients did not have a prior PTSD pathology. However, the presence of PTSD symptoms after trauma was not specifically identified and should be considered as a confounding variable.

According to self-reports, anxiety and depressive mood were not present before the trauma for the majority of the cohort studied (70\%) (see Table 1). After MTBI, a clear relationship was observed between level of anxiety and depression with those patients who were more anxious and also having a greater chance of being depressed. These psychological states seem to be a clear reaction to the traumatic event and/or the MTBI sustained. However, one might pose the question as to who is more vulnerable for developing an acute psychological reaction after MTBI, that is, who is more at risk. Some studies have shown that preinjury factors such as vocational status, lower educational level, and psychosocial difficulties including substance abuse $[5,12]$ and a preexisting mood disorder [3] could influence long term outcome of patients with MTBI. The current results corroborate the findings of the majority of work already published. In this investigation, the group with psychological antecedents developed a higher level of anxiety and depression after MTBI, and their cognitive performances were generally lower than the group without psychological antecedents. Moreover, both level of depression and anxiety were significantly higher for the divorced group compared to the single or married group. The anxiety score (but not the depression score) was significantly higher for those who were working before the trauma compared to those who were not working or who were students. It is possible that pressure from the employer to reintegrate work had an impact on workers and contributed to their anxiety level. The pressure could also be provided by the patient himself. Financial consequences were probably less of a concern for our subjects because a large number of them were being compensated for their injuries by governmental legislated insurance funds either for car accidents or for victims of criminal acts. In addition, the universal health system in Quebec covered all care provided by health professionals. Looking at other factors, anxiety level was not significantly different between the groups with and without a history of alcohol abuse, whereas the depression scores were significantly higher in those with prior alcohol problems. Moreover, anxiety level and depression at two weeks after MTBI were higher in the group with psychological antecedents compared to the group without. There was no difference however, between the groups with and without a significant medical history or the groups with or without a previous TBI for either anxiety or depression and no difference between men and women or for different levels of education. There was little variability in educational levels of the group, the majority of subjects having 7 years of schooling or more, so this could explain the lack of relationship with psychological reactions.

4.2. Clinical Impact and Conclusion. The acute higher level of anxiety and depression among patients with MTBI is an important finding of this study and should translate into specific resources attributed to service this clientele. The patients perceive their cognition as diminished even though neuropsychological scores remain within the "normative" zones. This perception leads to psychological distress. Clinicians and administrators need to consider offering psychological support by trained psychologists. Brief psychological therapy would probably be effective in reducing the intensity of perceived postconcussive symptoms and lead to a more favorable outcome. Moreover, intervention at the cognitive and psychological levels should focus more specifically on educating patients and clinicians about how sequelae at these levels influence each other after mTBI. As it would seem that psychological problems are more difficult to deal with than physical or cognitive problems following a TBI [42, 43], specific psychological intervention in the acute phase should be provided to patients with MTBI. The goal of this intervention targeting anxiety and depression at the acute level would be to decrease the risk of enduring long-term or even permanent symptoms.

\section{References}

[1] J. D. Cassidy, L. J. Carroll, P. M. Peloso et al., "Incidence, risk factors and prevention of mild traumatic brain injury: results of the WHO Collaborating Centre Task Force on Mild Traumatic Brain Injury," Journal of Rehabilitation Medicine, no. 43, pp. 28-60, 2004.

[2] L. J. Carroll, J. D. Cassidy, L. Holm, J. Kraus, and V. G. Coronado, "Methodological issues and research recommendations for mild traumatic brain injury: the WHO Collaborating Centre Task Force on Mild Traumatic Brain Injury," Journal of Rehabilitation Medicine, no. 43, pp. 113-125, 2004.

[3] J. Ponsford, C. Willmott, A. Rothwell et al., "Factors influencing outcome following mild traumatic brain injury in adults," 
Journal of the International Neuropsychological Society, vol. 6, no. 5, pp. 568-579, 2000.

[4] L. Chamelian and A. Feinstein, "The effect of major depression on subjective and objective cognitive deficits in mild to moderate traumatic brain injury," Journal of Neuropsychiatry and Clinical Neurosciences, vol. 18, no. 1, pp. 33-38, 2006.

[5] M. R. Hibbard, T. A. Ashman, L. A. Spielman, D. Chun, H. J. Charatz, and S. Melvin, "Relationship between depression and psychosocial functioning after traumatic brain injury," Archives of Physical Medicine and Rehabilitation, vol. 85, no. 2, pp. S43-S53, 2004.

[6] L. M. Binder and M. L. Rohling, "Money matters: a metaanalytic review of the effects of financial incentives on recovery after closed-head injury," American Journal of Psychiatry, vol. 153, no. 1, pp. 7-10, 1996.

[7] A. Lundin, C. de Boussard, G. Edman, and J. Borg, "Symptoms and disability until 3 months after mild TBI," Brain Injury, vol. 20, no. 8, pp. 799-806, 2006.

[8] M. R. Hibbard, S. Uysal, K. Kepler, J. Bogdany, and J. Silver, "Axis I psychopathology in individuals with traumatic brain injury," Journal of Head Trauma Rehabilitation, vol. 13, no. 4, pp. 24-39, 1998.

[9] J. S. Kreutzer, R. T. Seel, and E. Gourley, "The prevalence and symptom rates of depression after traumatic brain injury: a comprehensive examination," Brain Injury, vol. 15, no. 7, pp. 563-576, 2001.

[10] J. Ponsford, S. Sloan, and P. Snow, Traumatic Brain Injury: Rehabilitation for Everyday Adaptative Living, Lawrence Erlbaum Associates, Est Sussex, UK, 1995.

[11] J. F. Malec, J. A. Testa, B. K. Rush, A. W. Brown, and A. M. Moessner, "Self-assessment of impairment, impaired self-awareness, and depression after traumatic brain injury," Journal of Head Trauma Rehabilitation, vol. 22, no. 3, pp. 156166, 2007.

[12] S. S. Dikmen, C. H. Bombardier, J. E. MacHamer, J. R. Fann, and N. R. Temkin, "Natural history of depression in traumatic brain injury," Archives of Physical Medicine and Rehabilitation, vol. 85, no. 9, pp. 1457-1464, 2004.

[13] G. L. Iverson, "Misdiagnosis of the persistent postconcussion syndrome in patients with depression," Archives of Clinical Neuropsychology, vol. 21, no. 4, pp. 303-310, 2006.

[14] M. L. Rohling, P. Green, L. M. Allen, and G. L. Iverson, "Depressive symptoms and neurocognitive test scores in patients passing symptom validity tests," Archives of Clinical Neuropsychology, vol. 17, no. 3, pp. 205-222, 2002.

[15] D. E. Trahan, C. E. Ross, and S. L. Trahan, "Relationships among postconcussional-type symptoms, depression, and anxiety in neurologically normal young adults and victims of mild brain injury," Archives of Clinical Neuropsychology, vol. 16, no. 5, pp. 435-445, 2001.

[16] M. Stulemeijer, P. E. Vos, G. Bleijenberg, and S. P. van der Werf, "Cognitive complaints after mild traumatic brain injury: things are not always what they seem," Journal of Psychosomatic Research, vol. 63, no. 6, pp. 637-645, 2007.

[17] L. Holm, J. D. Cassidy, L. J. Carroll, and J. Borg, "Summary of the WHO Collaborating Centre for neurotrauma Task force on mild traumatic brain injury," Journal of Rehabilitation Medicine, vol. 37, no. 3, pp. 137-141, 2005.

[18] J. Borg, L. Holm, J. D. Cassidy et al., "Diagnostic procedures in mild traumatic brain injury: results of the WHO Collaborating Centre Task Force on Mild Traumatic Brain Injury," Journal of Rehabilitation Medicine, no. 43, pp. 61-75, 2004.

[19] J. Corrigan, J. Borgner, and G. Lamb-Hart, "Problematic substance use identified in the TBI Model Systems national database," The Center for Outcome Measurement in Brain Injury, November 2005, http://tbims.org/combi/subst/index .html.

[20] N. S. King, S. Crawford, F. J. Wenden, N. E. G. Moss, and D. T. Wade, "The Rivermead post concussive symptoms questionnaire: a measure of symptoms commonly experienced after head injury and its reliability," Journal of Neurology, vol. 242, no. 9, pp. 587-592, 1995.

[21] A. T. Beck and R. A. Steer, Manual for the Beck Anxiety Inventory, Psychological Corporation, San Antonio, Tex, USA, 1990.

[22] A. T. Beck, C. H. Ward, M. Mendelson, J. Mock, and J. Erbaugh, "An inventory for measuring depression," Archives of General Psychiatry, vol. 4, pp. 561-571, 1961.

[23] H. Hooper, Hooper Visual Organization Test (HVOT), Western Psychological Services, Los Angeles, Calif, USA, 1983.

[24] L. Gauthier, F. Dehaut, and Y. Joanette, "The bells test: a quantitative and qualitative test for visual neglect," International Journal of Clinical Neuropsychology, vol. 11, no. 2, pp. 49-54, 1989.

[25] D. Wechsler, WMS III Manual, The Psychological Corporation, New York, NY, USA, 1997.

[26] D. Wechsler, WAIS-III Manual, The Psychological Corporation, New York, NY, USA, 1997.

[27] P. S. Osterrieth, "Le test de copie d'une figure complexe," Archives de Psychologie, vol. 30, pp. 206-356, 1944.

[28] J. Brandt, "The Hopkins verbal learning test: development of a new memory test with six equivalent forms," Clinical Neuropsychologist, vol. 5, no. 2, pp. 125-142, 1991.

[29] O. Spreen and E. Strauss, A Compendium of Neuropsychological Tests, Oxford University Press, New York, NY, USA, 1991.

[30] R. M. Reitan, "Validity of the trail making test as indicator of organic brain damage," Perceptual and Motor Skills, vol. 8, pp. 271-276, 1958.

[31] M. Creamer, J. Foran, and R. Bell, "The Beck anxiety inventory in a non-clinical sample," Behaviour Research and Therapy, vol. 33, no. 4, pp. 477-485, 1995.

[32] J. M. Pomerantz, "Screening for depression in primary care," Drug Benefit Trends, vol. 17, no. 6, pp. 273-274, 2005.

[33] E. D. Bigler and M. Brooks, "Traumatic brain injury and forensic neuropsychology," Journal of Head Trauma Rehabilitation, vol. 24, no. 2, pp. 76-87, 2009.

[34] N. Gosselin, C. Bottari, J. K. Chen et al., "Electrophysiological and functional MRI in post-cute mild traumatic brain injury," Journal of Neurotrauma, vol. 28, pp. 329-341, 2011.

[35] N. Garden and K. A. Sullivan, "An examination of the base rates of post-concussion symptoms: the influence of demographics and depression," Applied Neuropsychology, vol. 17, no. 1, pp. 1-7, 2010.

[36] P. Rabbitt and V. Abson, "Lost and found: some logical and methodological limitations of self-report questionnaires as tools to study cognitive ageing," The British Journal of Psychology, vol. 81, pp. 1-16, 1990.

[37] P. Nolin, R. Villemure, and L. Heroux, "Determining longterm symptoms following mild traumatic brain injury: method of interview affects self-report," Brain Injury, vol. 20, no. 11, pp. 1147-1154, 2006.

[38] B. Ravnkilde, P. Videbech, K. Clemmensen, A. Egander, N. A. Rasmussen, and R. Rosenberg, "Cognitive deficits in major depression," Scandinavian Journal of Psychology, vol. 43, no. 3, pp. 239-251, 2002.

[39] N. I. Landrø, T. C. Stiles, and H. Sletvold, "Neuropsychological function in nonpsychotic unipolar major depression," 
Neuropsychiatry, Neuropsychology and Behavioral Neurology, vol. 14, no. 4, pp. 233-240, 2001.

[40] J. F. Malec, A. W. Brown, A. M. Moessner, T. E. Stump, and P. Monahan, "A preliminary model for posttraumatic brain injury depression," Archives of Physical Medicine and Rehabilitation, vol. 91, no. 7, pp. 1087-1097, 2010.

[41] S. J. Lupien, F. Maheu, M. Tu, A. Fiocco, and T. E. Schramek, "The effects of stress and stress hormones on human cognition: implications for the field of brain and cognition," Brain and Cognition, vol. 65, no. 3, pp. 209-237, 2007.

[42] M. Rosenthal, B. K. Christensen, and T. P. Ross, "Depression following traumatic brain injury," Archives of Physical Medicine and Rehabilitation, vol. 79, no. 1, pp. 90-103, 1998.

[43] M. V. Morton and P. Wehman, "Psychosocial and emotional sequelae of individuals with traumatic brain injury: a literature review and recommendations," Brain Injury, vol. 9, no. 1, pp. 81-92, 1995. 


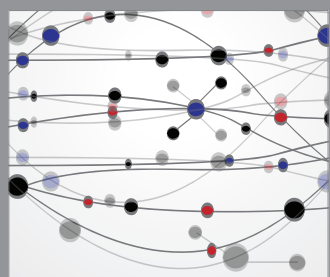

The Scientific World Journal
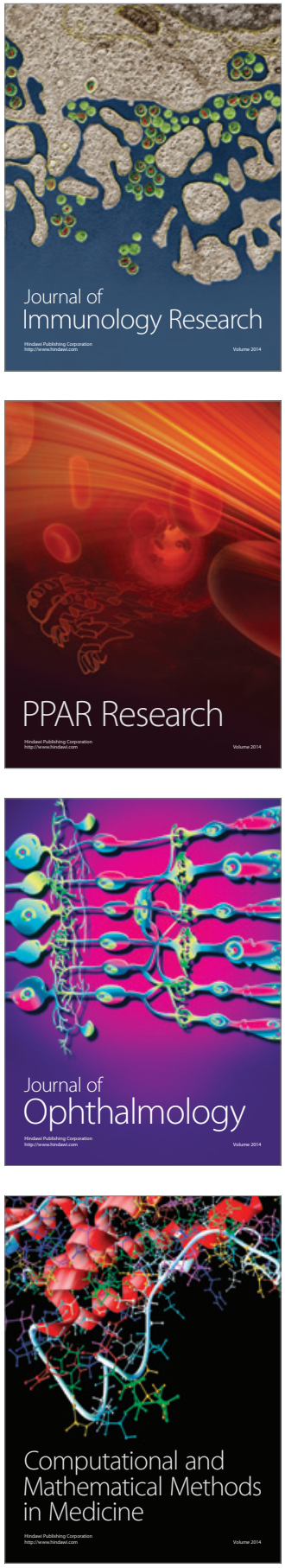

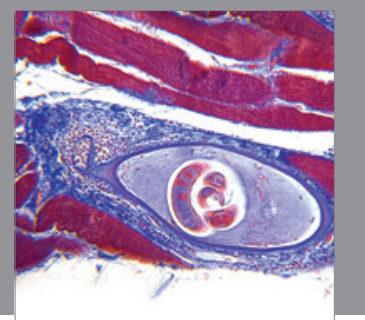

Gastroenterology

Research and Practice
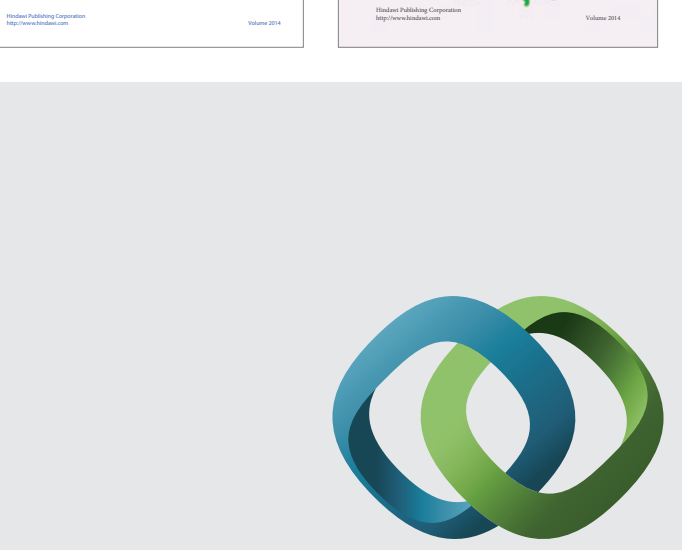

\section{Hindawi}

Submit your manuscripts at

http://www.hindawi.com
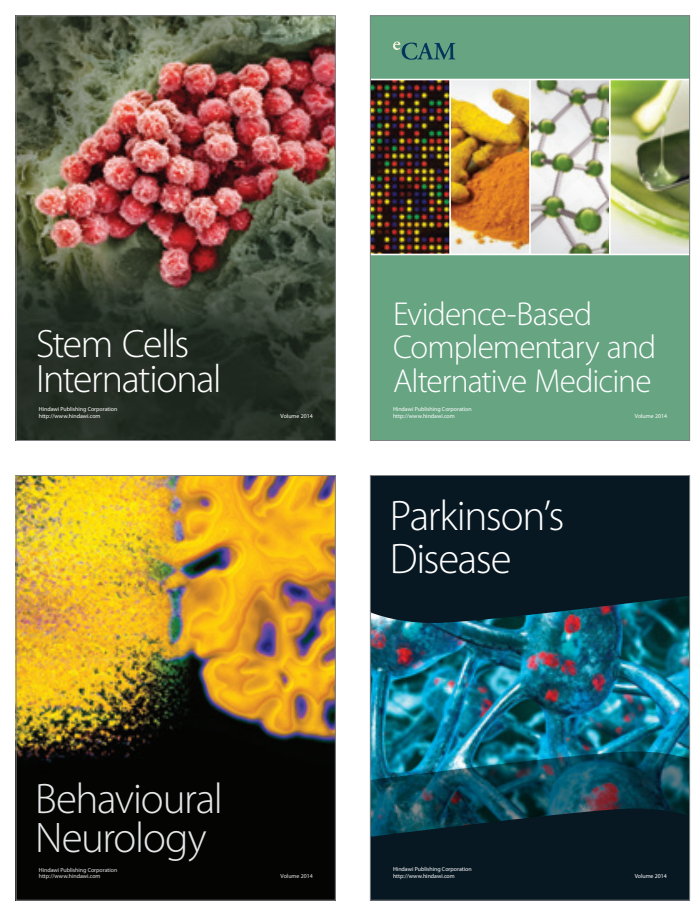

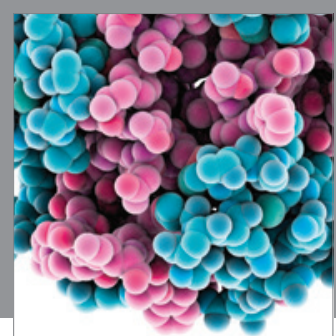

Journal of
Diabetes Research

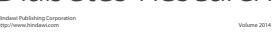

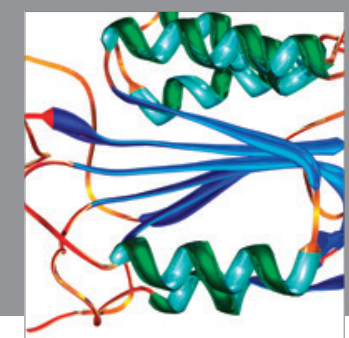

Disease Markers
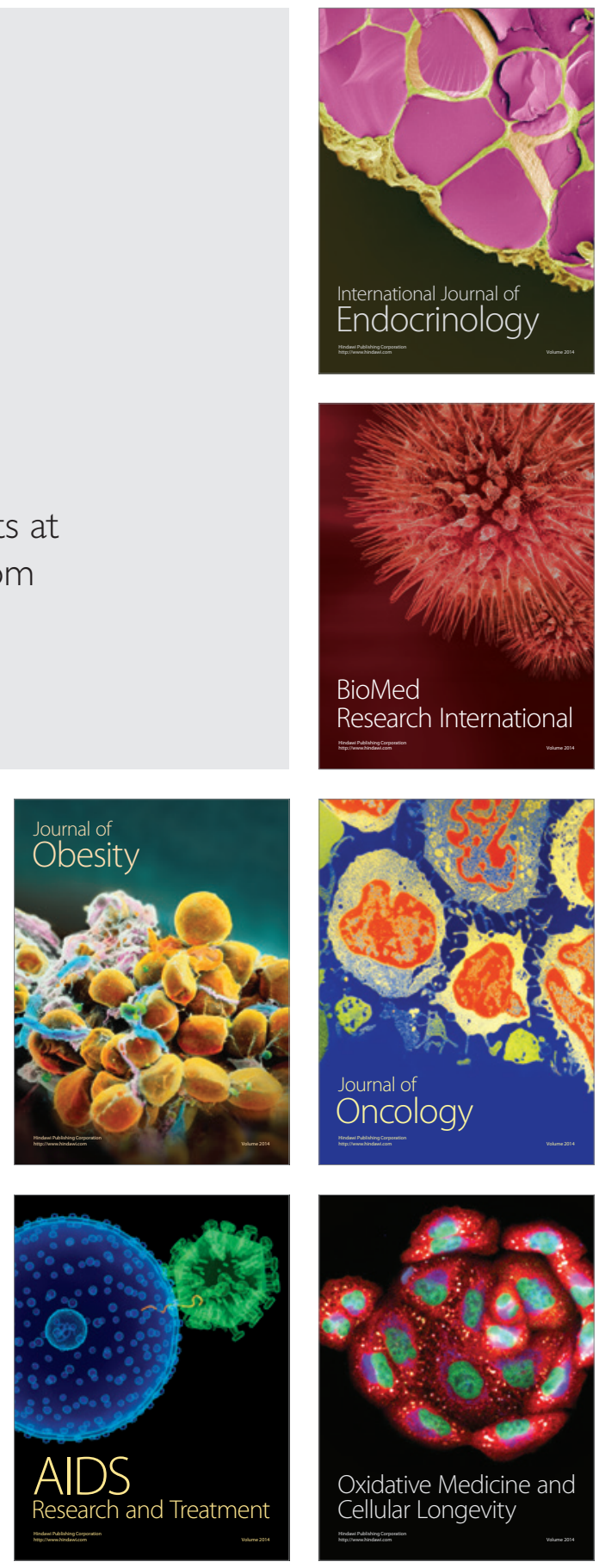